\title{
Rationalising Rule Violation in the Case of the Chernobyl Disaster: Six Systematic Excuses
}

This paper investigates how rule violations that contributed to the Chernobyl nuclear disaster were able to happen. Bringing to the surface what is implicit in historical documents, we argue that six excuses can be identified that could have allowed the operators to rationalise their rule violations. These excuses could have affected how the operators interpreted the applicability of the rules to the situation(s) they were involved in, leading them to ultimately violate the operational rules. As a theoretical consequence, rule-following as (com)pliance or obedience is to be taken as a necessary but insufficient condition of rule-following. As a practical consequence, the concept of 'excuses' can also help to anticipate as well as prevent rule-breaking behaviour in similar future cases.

Keywords: Chernobyl nuclear disaster, rule-following behaviour, actual rule violation, negligent rule violation, rationalisation

\section{Acknowledgement}

We are grateful to Ákos Horváth, Director General of the Hungarian Academy of Sciences, for his help and consultation. We would also like to thank Tihamér Margitay for his valuable comments and Ákos Gyarmathy for the inspiration to start the research on the present topic.

\section{Author Information}

István Danka, Budapest University of Technology and Economics https://orcid.org/0000-0002-8946-2556

János Tanács, Eötvös Loránd University https://orcid.org/0000-0003-4653-4991

How to cite this article:

Danka, István, János Tanács. "Rationalising Rule Violation in the Case of the Chernobyl Disaster: Six Systematic Excuses”. Információs Társadalom XXI, no. 4 (2021): 19-37. 


\section{Introduction}

The International Nuclear Safety Advisory Group (INSAG) of the International Atomic Energy Agency (IAEA) published a second report in 1992 into the Chernobyl Nuclear Power Plant accident (INSAG-7 1992). This second report, INSAG-7, re-evaluated in many ways the first one made in 1986 (which is often referred to as the INSAG-1 report). The most significant changes included the revelation of some design flaws. These flaws became accepted as the root causes of the accident. In the second report, there was also a change in the perception of irregularities committed by the operators: the INSAG-7 report essentially took the burden of non-compliant behaviour off the shoulders of operators and shifted the blame for the accident mainly to design flaws (INSAG-7 1992, 24). According to INSAG-7, with one exception, the violations identified in the first report were either not actually infringements in the absence of relevant provisions (INSAG-7 1992, 24; Szathmáry and Aszódi 2005, 94) or played no role and had no significance in causing the accident, because either their observance would not have prevented the accident or would not have reduced its severity.

Among others, a construction flaw was identified in the design of the control and safety rods; whereby, when a fully withdrawn rod was inserted into the core, an initial positive reactivity occurred.

Due to this effect, the control and safety rods could not decrease the rate of the chain reaction from the very first moment of the insertion but actually increased the rate for a while. The initial insertion of positive reactivity from control and safety rods, when dropped into the core from the fully withdrawn position, is known as the positive scram effect and occurred at Chernobyl due to a deficiency in the design of the reactor and in the rods in particular. The phenomenon of the positive scram effect made the emergency system "not only ineffective but also destructive" (INSAG-7 1992, 18).

Immediately before the accident, 203-205 rods out of the 211 were fully withdrawn. Consequently, when the EPS-5 emergency button was pressed at 01:23:40 on 26 April and the rods were dropped into the reactor, that number of rods was able to insert a serious amount of positive reactivity in the lower half of the core. Therefore, the effect of inserting too many fully withdrawn rods was assessed as "probably a decisive contributory factor" in causing or exacerbating the accident (INSAG-7 1992, 23). The insertion of "too many fully withdrawn rods" implied too low an operating reactivity margin (ORM). The importance of this was seriously underestimated. INSAG-7 ultimately stated that "operation with too low an ORM was a violation of requirements” (INSAG-7 1992, 18).

The operating reactivity margin (ORM) is defined as "the extra reactivity that would arise if all control and safety rods were withdrawn, expressed as a multiple of the total reactivity controlled by a standard rod" (INSAG-7 1992, 6). "Operation with too low an ORM" in the report meant going below the allowed absolute minimum of 15 rods. Under that value, the regulations implied the unit should be immediately shut down. Although the second report did not consider the violation of the ORM to be an initiating cause of the accident, "the scram just before the sharp rise in power that destroyed the reactor may well have been the decisive contributory factor" (INSAG-7 1992, 13). 
While INSAG-1 identified six violations as major causes of the accident (INSAG-7 1992, 17), INSAG-7 accepted only one direct violation out of the formerly identified six, namely the "operation with too low an ORM". But even in that case, it was claimed that the violation had been "important for different reasons from those previously understood" (INSAG-7 1992, 18). So, the second report also reconsidered the status of non-compliance, since it no longer claimed that ORM had played a root cause, even if it left this in as the only one of the six factual violations committed by the operators initially identified by the IAEA.

Since the ORM violation was a factual as well as decisive contributory factor in the accident, the second report of the Advisory Group paid more attention to it, making some reflections on the details that could have played a role in the rule-breakings. This allowed us to investigate what the IAEA considers implicitly as important factors concerning the rule-(un)following behaviour.

This paper consists of six sections. After this introductory section, Section 2 provides the background to the safety culture or safety management and links rule-compliance with the views expressed in the IAEA documents. Section 3 analyses the remarks in INSAG-7 on ORM rule violations prior to the accident and provides theoretical grounds for the idea that rule-following as (com)pliance must be exceeded in order to explain the situation. Section 4 reconstructs the cases of ORM rule violations as time progressed towards the accident, identifies the work shifts involved in the violation(s), and categorises the types of rule violations that occurred. Section 5 deduces six 'excuses' from INSAG-7 and further historical resources. These excuses are helpful for conceptualising the factors that could have contributed to the rule violations. Section 6 generalises the consequences beyond the Chernobyl case and also offers directions towards possible solutions for avoiding similar problems occurring in future cases, whenever complex rule-following beyond pure (com)pliance or obedience occurs.

\section{Rule Violation and Safety Culture}

The issue of rule violation is discussed somewhat indirectly in the IAEA papers or INSAG reports. These documents address the issue of compliance as part of safety culture or safety management. INSAG-15 (referring to INSAG-11) states that the organisations behind nuclear plants should go through three phases in developing and strengthening the safety culture (INSAG-15 2002, 2). Stage 1 is when safety is compliance-driven or regulation-based. Stage 2 is when "good safety performance becomes an organizational goal": safety is based on a goal-driven mode of operation. In Stage 3, "safety is seen as a continuing process of improvement", so it is a kind of contribution-based organisational operation.

Therefore, the issue of rule compliance and rule violation is addressed here in the context of Stage 1. In this stage, safety is basically and almost exclusively a technical issue, and "compliance with externally imposed rules and regulations is considered adequate for safety” (INSAG-15 2002, 2). Although the most desirable stage to be in is Stage 3, to reach this level, the former two cannot be skipped or left behind 
too early, because "achieving good safety performance requires a rule-based compliance culture” (INSAG-15 2002, 4) to be developed at the earlier stages.

At the same time, the mode of operation expected in Stage 1 seems to be the most problematic among the three. On the one hand, compliance with regulations, rules and procedures is obviously important for safety (IAEA-TECDOC-1329 2002, 11), but on the other hand, at this stage usually "an organization sees safety as an external requirement, and not as an aspect of conduct that will allow it to succeed" (IAEA-TECDOC-1329 2002, 17). As compliance with regulations is seen to be a technical issue and considered an external requirement demanded by the regulatory bodies, "there is little awareness of the behavioural and attitudinal aspects of safety" (IAEA-TECDOC-1329 2002, 17).

However, Stage 1 has two important purposes. First, it must be fulfilled on its own. Second, it prepares for and supports the transition to Stage 2. The problem is that "an organization might possess characteristics associated with each of the three stages" (IAEA-TECDOC-1329 2002, 19), but the characteristics associated typically with Stage 1 may not support the transition from Stage 1 to Stage 2. In order to understand this problem, let us note some typical characteristics of an organisation that is in Stage 1 (IAEA-TECDOC-1329 2002, 18-19):

- People who make mistakes are blamed for their failure to comply with the rules.

- People are viewed as components of the system - the 'mechanistic view'.

- People are rewarded for obedience and results, regardless of the long-term consequences.

- The role of management is to enforce the rules.

In guidance meant to enhance the safety culture, the IAEA emphasises that:

... a rule-based approach should not be viewed negatively. There will be activities or circumstances in organizational life where strict compliance with rules is essential, e.g. emergency response, or operating with sufficient margin for safety. Cultural awareness is not incompatible with having strict rules; after all, much of culture is about complying or conforming to norms (IAEA-TECDOC-1329 2002, 19).

Taking the above as a framework, our analysis can be considered as follows. A rule-based approach is essential and indispensable in Stage 1, but the circumstances of rule-compliant behaviour as well as the wording and the communication of the rules and regulations must serve as a preparatory stage for the next one. To achieve the latter goal, some problematic or negative characteristics of Stage 1 must be avoided. For example, operators cannot be viewed as components of the system, or rule-compliant behaviour cannot be viewed mechanistically. Rule-following agents are mostly supposed to be rational, but fallible. 'Being rational' in this context means that rule-compliant behaviour must be seen as an intellectually driven behaviour, whereas 'being fallible' means that there are circumstances and factors that can strengthen or weaken the willingness of rule-compliant behaviour. If safety is based on a goal-driven mode of operation as in Stage 2, a mechanistic view is inapplicable 
also, insofar as goal-drivenness cannot be grounded purely in compliance with the rules as means.

Our approach is akin to that of Abramova (Abramova 2019, 81-100) who introduced a classification of the incorrect actions of nuclear power plant staff based on the causes of those incorrect actions (Abramova 2019, 95-97). The causes identified by Abramova are heterogenous in a sense that there are external causes as well as internal ones: some of the causes are rooted exclusively in the agents, while others are rooted in the operating conditions. Where our approach differs from Abramova's is that we focus on the operating conditions and circumstances in order to identify their roles in the willingness of rule-compliant behaviour by agents. So, our classification captures the 'excuses' rather than the causes, such as the internal or intellectual transformation of external factors.

\section{Reflections on the Violations of the ORM Regulation in the INSAG-7 Report}

The second report of the IAEA made the following observation concerning the regulation of ORM. First, in a section on the Operating Procedures, the reactivity, in general, was referred to as an important operating parameter, but the ORM as a special parameter was not included in the list of important parameters (INSAG-7 1992, 72). Second, there was no device available to measure the ORM simply and effectively. Although the operators had two ways to measure the ORM, both were complicated. According to the first way, the operator had to determine the depth of the rods in the core, then correct the results for the non-linearity of the graduation scale, and finally to sum them up. The second way was to instruct the plant computer to receive data and to make the necessary calculations with the PRIZMA program. Both were time-consuming since it took 10-15 minutes to get the actual ORM value either way. The location of the device to measure the ORM also further complicated the process, since it was held approximately 50 metres away from the control console of the operators (INSAG-7 1992, 6, 72). These are the reasons why the report stated that "it seems unreasonable to expect the personnel to treat this parameter [ORM] as a directly controllable one” (INSAG-7 1992, 72).

The third point in the reflections of the report claims that "the Operating Procedures did not draw the attention of the personnel to the importance of the ORM as an essential parameter for ensuring the effectiveness of the emergency protection system" (INSAG-7 1992, 72). This observation means that the Operating Procedures interpreted the ORM as merely a manoeuvring parameter to keep the power distribution balanced throughout such a large and loosely coupled core as that of Unit 4 at Chernobyl NPP, but not a parameter that was important for ensuring the effectiveness of the emergency protection system (INSAG-7 1992, 14, 72). Due to the one-sided and incomplete interpretation of the ORM, the operators were not aware of the emergency aspect of the ORM.

These reflections are important for several reasons. First, INSAG-7 implicitly acknowledges that a naïve or trivial conception of rule-following is insufficient to 
understand the operators' situation in which they must follow the rules. This insufficiency results in their not being instructed properly to follow the rules. Second, rule-following as pure (com)pliance or obedience is not sufficient for making agents follow rules unconditionally. Third, some factors can strengthen or weaken the intention of rule-followers to follow the regulation in a particular situation. So, in the following sections, following the spirit of INSAG-7, we further investigate the factors that could help, or hinder, rationalising rule-breaking from the subjective perspective of the agent. In order to explore the situation of the ORM violation in a more nuanced way, let us first overview the violations of ORM committed during the 24hour preparation process leading up to the fatal test.

\section{History of the Violations of the Regulations for the Operating Reactivity Margin During the Two-day Preparation for the Turbogenerator Test}

The events that led to the accident at Unit 4 of the Chernobyl Nuclear Power Plant at 1:24 on the 26th of April, 1986, started on the 25th of April at 1:06. There was a test scheduled to examine the stability of the operation of the reactor in the event of the loss of the external power sources: this was the so-called turbogenerator test. As a first step in the preparation to carry out this test, the reactor power was gradually reduced to $50 \%$ of its nominal power. At 3:47, the reactor was stabilised at a thermal power of 1600 MW. Decreasing the reactor power so significantly in such a short time resulted in a temporary increase in the xenon content. Since xenon is a neutron absorbent, its increase resulted in a reduction of the reactivity. The effect of a reduction of the reactivity caused by a temporary increase in the xenon content is called 'xenon poisoning'. To counter this, the operators had to withdraw some control rods to increase the reactivity in the core in order to compensate for the effect of the xenon poisoning. A continuous withdrawal of the control rods led to a continuous decrease in the operating reactivity margin (ORM).

According to the operating log, the ORM was equivalent to 13.2 standard control rods at 7:10 on the 25th of April (INSAG-7 1992, 53). Section 9 of the Operating Procedures of Unit 4 nailed down what to do when the ORM decreases to 15 rods (or below): "When the ORM falls to 15 rods, the reactor should be shut down immediately" (INSAG-7 1992, 79).

However, the value of the ORM was less than 15 rods for a period between 6:00 and 9:00 on the 25th of April. This was the case when Igor Kazachkov, head of the day shift (8:00-16:00) took over the reactor from the dawn shift. Kazachkov later stated that he and his colleagues were aware of the regulation that operating below the value of 15 ORM rods was forbidden, and that the reactor should have been shut down immediately (Plokhy 2018, 90-91).

In the following step of the preparation, at 14:00, the emergency core cooling system (ECCS) was disconnected from the multipass forced circulation circuit (MFCC) (INSAG-7 1992, 53; Szathmáry and Aszódi 2005, 83), but the Kyiv power grid controller asked to postpone the test programme at that time (INSAG-7 1992, 53; Plokhy 
2018, 93). According to the original schedule, the turbogenerator test, as well as the shutdown of the reactor for the scheduled medium-term maintenance, should have been finished by 16:00 on the 25th of April.

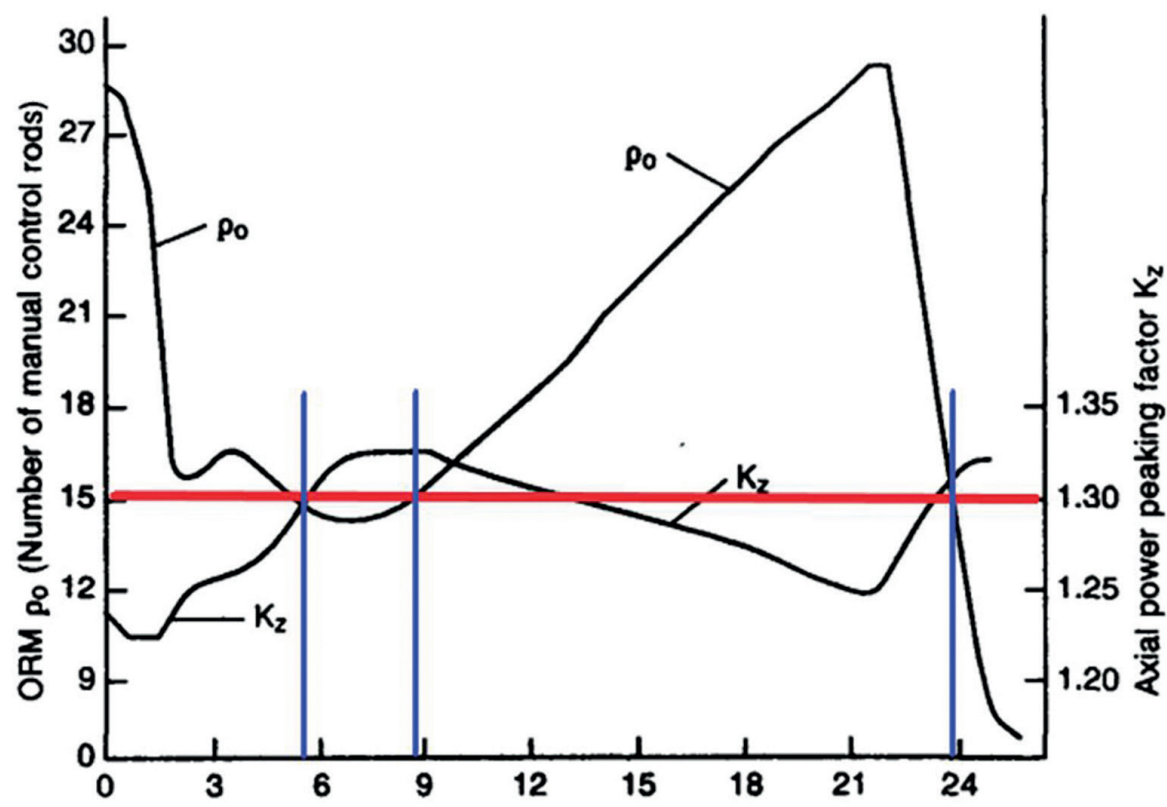

Figure 1. Change in the operating reactivity margin $(\rho 0)$ from the beginning of the preparation of the turbogenerator test till the time of the accident $(t=0$ is $1: 06,25$ th of April). The horizontal red line indicates the minimum level of ORM $=15$. The vertical blue lines indicate the periods when the ORM was less than 15 rods. Figure taken from INSAG-7 $(1992,117)$; the red and blue lines were added by us.

The value of the ORM was 16.8 at 15:20, so it was above the prescribed minimum number of 15 rods (INSAG-7 1992, 53). The Kyiv power grid controller permitted disconnecting Unit 4 from the power grid at 23:10. The ORM was 26 rods at that time. Then, a rapid power reduction started again at 23:10. The operators had to withdraw a serious number of control rods to compensate for the reduced reactivity caused by the xenon poisoning. The continuous withdrawal of the control rods resulted in a constant decrease in the ORM.

At $00: 28$ on the 26th of April, when the local automatic control system was switched over to the global one, a sudden and unexpected event occurred: the thermal power of the reactor fell from $500 \mathrm{MW}$ to approximately 0-30 MW (INSAG-7 1992, 112; Szathmáry and Aszódi 2005, 83). This was a kind of unintentional shutdown and the operators did not have an explanation for the incidence. In order to increase the power after the non-envisaged performance drop, the operator had to withdraw more control rods from the reactor core.

From this moment, the events can be reconstructed in two different ways. According to the first version, there was no entry regarding the value of the ORM in 
the operating log after 23:10 on the 25th of April, so the operators did not know the value of the ORM during the critical part of the test (INSAG-7 1992,11). Not long before the test started at 1:23:10 on the 26th of April, the ORM was significantly lower than the 15 rods allowed: it was approximately 8 rods at 1:22:30 (INSAG-7 1992, 53). According to this historical reconstruction, the operators did not know the value of the ORM of that time; its value was calculated only after the accident (INSAG-7 1992, 113). The second historical reconstruction suggests that at the beginning of the test at 1:23:10, the operators knew the value of the ORM measured at 1:22:30, but not the importance of violating the ORM regulation this way (INSAG-7 1992, 79; Malko 2002, 19).

Taking all this into consideration, it can be stated that during the 24-hour preparation period for the test, the operators violated Section 9 of the Operating Procedures concerning the regulation of the minimum allowable operating reactivity margin several times. The ORM was constantly lower than the allowed minimum during two periods in the 24-hour preparation time. The first violation period was between 6:00 and 9:00 on the 25th of April, and two shifts were involved in the violation. The second violation period lasted from midnight of the 25th of April till the accident itself (INSAG-7 1992, 116-117), (see also Fig. 1).

During the first violation period, a factual, constant rule-breaking happened, and the operators were aware of the violation of the regulation. During the second violation period, the rule-breaking could be categorised into two different types: If the operators knew the ORM measured at 1:22:30, then the rule-breaking was also factual, but if the ORM was calculated only after the accident for the investigation committee, then the rule-breaking could be considered as a negligent violation.

A negligent violation is a case when a person is expected to follow a rule, and therefore he or she is also expected to obtain all the relevant information that is required for following a rule, but in fact, he or she does not obtain some or all the necessary pieces of this information. According to the first historical version, this was exactly the case during the second violation period: the operator had to withdraw control rods constantly in order to compensate for the temporal xenon poisoning, which was due to the further reduction in the power level initiated at 23:10. Even though they knew the regulation that the unit was to be shut down when the ORM went below 15, lacking making an effort to get the ORM value made their behaviour a negligent violation. If the first historical reconstruction is right, the operators did not know all the relevant information (they did not know the actual ORM value) during the second violation period, but they should have known that value to ensure they could operate the reactor properly, and they could have got the value (even if it was not easy to get). But this was not a direct, factual violation, since a direct, factual violation means that the rule-breakers know all the relevant information and despite that, they still fail to follow a rule.

All in all, it can be said either that four shifts in two violation periods committed the actual violations of the minimum permitted ORM, or two shifts committed factual violations and two committed negligent ones. 


\section{Conceptualising the Factors Contributing to the Rule Violations as Possible 'Excuses'}

From the reconstruction of the rule violations based on INSAG-7 (1992) and consideration of the further historical resources provided above, six factors can be identified as relevant for the rule violations. We shall call these 'excuses', because as far as it can be said on the grounds of historical evidence, they could serve as subjective excuses for the operators violating the ORM rules. These excuses are as follows. First, the reactor was claimed to be fail-safe, and second, similar rule-violations happened regularly with no consequences. These two together made the operators think (as far as it can be seen from the reports) that there was no chance of an accident: the rules could be violated with no negative consequences if the reactor was fail-safe and rule violations had also happened earlier with no negative consequences. There was also an accessibility problem with the ORM values: due to the third excuse, the ORM values were hardly accessible and, due to the fourth, occasionally they could even be temporarily inaccessible. In the case of the lack of proper accessibility, they could not be measured easily or temporally, leading to a negligent violation of the ORM rules. Fifth, the ORM values were not included in the important parameters' list; implicitly suggesting that they were not important. Sixth, the emergency protection functions of the ORM were unknown to the operators, further decreasing the apparent negligibility of the relevant rules, especially in emergencies in which obeying them would have been crucial.

Hence, relations can be drawn among these excuses as follows. The first and second excuses together served as grounds for thinking that there was no chance of an accident. The third and the fourth excuses built on the problems of the accessibility of relevant information for operating the power plant properly (namely, the ORM values), resulting in potential negligent rule violation(s). Finally, the fifth and sixth factors served as reasons prima facie supporting that the ORM values were unimportant. Note that each excuse could serve as a prima facie reason for the operators to break the regulations, and together they mutually strengthened the inclination.

In the following sections, we investigate how these excuses contributed to the operators' inclination to violate the ORM rule, partly based on the grounds of their personal reports and partly on historical documents and reconstructions.

Before turning to the excuses in detail, let us make some clarifications. Our goal is not to absolve the operators. Whether they had subjectively 'good' reasons for the rule violation or not it does not undo the rule violations and their consequences. We are not interested in their moral responsibility or accountability either. Our goal is to understand the (actual or possible) reasons for the rule violations that happened in Chernobyl, which can contribute to an account of the rule-following/ violating behaviour on the one hand, and, on the other, it can also help instructors and rule-makers prevent similar reasoning, and hence possible similar accidents, in the future. 


\section{1. "No chance of an accident"}

The false belief of the operators that there was no chance of an accident was built on two premises: first, the reactor was designed and communicated to be fail-safe, and second, based on inductive reasoning, if earlier (similar) rule violations had not had consequences, further violations would not have consequences either. The first preceded, but was also constantly supported by, the second, as the second made the first (couple of) rule violations excusable. On the grounds of the first couple of rule violations, which had not entailed any empirical or operating consequences, inductive reasoning could start to work in the background, suggesting that the rule could be violated without consequences. So, the myth of the fail-safety and the inductively confirmed experience of breaking the rule without any consequences contributed to the operators' inclination to violate the ORM rules. In any case, the myth of fail-safety and induction could go hand in hand, increasing one another's strength.

Let us investigate the role of these factors one by one.

\subsubsection{The myth of fail-safety}

Both INSAG-1 and INSAG-7 claimed that "the primary cause of the accident was an extremely improbable combination of violations by the unit personnel of operating instructions and procedures" (INSAG-7 1992, 30). The circumstances of the Chernobyl disaster were extremely sensitive to the prestige and reputation of the Soviet Union, and this can be an explanation why INSAG-1 emphasised personal faults and "extreme improbabilities" only. In 1992, the year after Gorbachev's resignation and the dissolution of the Soviet Union, INSAG-7 indicated that mistakes in the design and instructions also played a significant role in the disaster. "The weight given in INSAG-1 in 1986 to the Soviet view [...], which laid blame almost entirely on actions of the operating staff, is thereby lessened" (INSAG-7 1992, 24).

The Soviet nuclear programme, as well as the RBMK-type reactor, were taken as symbols of Soviet supremacy over the US (which itself had suffered a loss of reputation regarding nuclear energy safety due to the Three Miles Island accident in 1979 that led to a decline in the expansion of nuclear power plants (Hultman and Koomey 2013)). But the protection of reputation was not the only explanatory factor of why the unit personnel were claimed to be responsible: the myth of fail-safety for the RMBK-type nuclear reactors also prevented an acknowledgement of the design deficiencies. When in 1986, Gorbachev was informed about the explosion accompanied by the nuclear fission emission, his first reaction was amazement, asking how it could have taken place. "After all, the scientists had always assured us, the country's leaders, that the reactor was absolutely safe”, he recalled (Plokhy 2018, 148).

One of these scientists was Anatoly Aleksandrov, the principal designer of the RBMK reactor, who "kept telling everyone who would listen that his reactors were safe and sound" (Plokhy 2018, 35). He also spread that "the RBMK reactor could be installed even on Red Square, since it was no more dangerous than a samovar" (Plokhy 2018, 148.) This slogan spread so successfully that plant managers, according to Legasov, echoed Aleksandrov's saying, claiming that "an atomic reactor is a 
samovar. It's much simpler than a thermal station, our personnel are experienced, and nothing ever happens” (Medvedev 1990, 369).

The myth of fail-safety was so strong that it ultimately led to denial. Even when the accident actually happened, the operators did not believe for quite some time that it could be a nuclear disaster since they were convinced that "the reactor and its panoply of safety systems were idiot-proof” (Plokhy 2018, 107-108).

So, it was widely believed and communicated nationwide that the reactor was fail-safe, and there was a common belief among operators that the Chernobyl reactor was absolutely safe and there was no chance of an accident at all. In light of these beliefs, it is much less surprising what beliefs the INSAG-7 report attributed to the operators about the positive scram effect of the rods:

Apparently, there was a widespread view that the conditions under which the positive scram effect would be important would never occur [italics added]. However, they did appear in almost every detail in the course of the actions leading to the accident (INSAG-7 1992, 13).

The myth of fail-safety had two important roles. First, it could support the first intentional violation of the rule of the minimum ORM. Second, it could support the interpretation of the results of the repeated rule-breaking.

Violations can happen either accidentally (when one does not know that they are violating a rule but comes to know that later only) or intentionally. Whether the first violation of the ORM rules was accidental or intentional is not known. It is worth examining both assumptions. First, let us suppose that it was accidental. When the operators realised the violation later, their belief that the reactor was failsafe was reinforced, even if the rules were violated accidentally. This could support their reaction in not preventing future occurrences but rather ignoring them. In this case, inductive reasoning, consciously or unconsciously, by the operators gradually strengthened the "no chance of an accident" excuse by corroborating the fail-safety excuse.

Second, if the first violation(s) had been intentional, the operators would not have had any previous examples of a violation, so their reasons could not have been inductive. Their potential excuse for violation could have been only the belief that the reactor was fail-safe. Then they had the first (few) individual case(s) as grounds for inductive reasoning. Hence, on this horn, the fail-safety myth served directly as a grounds for the first intentional violation(s).

\subsubsection{Inductive corroboration of rule-breaking without consequences}

Now let us focus on the inductive excuse for rule-breaking. After the first (few) violations, the rule-breakers had some inductive grounds for further breaking the rule. As said, rule-breaking was not unique in the Chernobyl case: even within the 24hour preparation for the test, the ORM rules were violated several times. The operators' previous experience suggested that there could be no accident because they just repeated operations they had done regularly. Hence, following simple inductive 
reasoning, they prima facie reasonably supposed, consciously or unconsciously, that no problem could occur if they did the same as they had done before.

From the fact(s) that one violated a rule in $\mathrm{t} 1, \mathrm{t} 2, \mathrm{t} 3, \ldots$, tn with no negative consequences, it follows inductively that in $\mathrm{tn}+1$, it is improbable that negative consequences would occur (the greater $\mathrm{n}$, the more improbable a negative consequence would be). The number of rods had gone below 15 several times earlier with no undesirable effects, so the operators prima facie reasonably believed that even though the regulations claimed going below ORM = 15 was forbidden, if it had gone that way all the same, nothing would happen, and they could continue the operation.

After some violations, the myth of fail-safety further supported the inductively corroborated belief that breaking the rule had no consequence. Since the no-consequence experience was in accordance with the belief of fail-safety, the latter could help to interpret the former. So, the inductively corroborated no-consequence experience and the myth of fail-safety mutually strengthened one another.

\subsection{The accessibility excuses}

Violating the ORM rule was therefore not without precedent. However, according to one of the historical reconstructions mentioned above, the violation was not actual but rather negligent at the time of the accident: the operators did not know the actual ORM value (INSAG-7 1992, 113). As said, in this case, the violation that happened should be labelled as a negligent violation: not obtaining the relevant information required for following a rule is an omission insofar as the information could have been accessed. Even though this type of violation is not a factual one, it highlights the importance of the conscious character of rule-following and the role of understanding the function as well as the content of the instructions. Let us look at the negligent type of violation in detail.

It can be supposed that the operators did not know the actual ORM value at the time of the accident. That being said, in the morning of the previous day of the accident, the ORM was measured as it went to ORM $=13.2$, and they did know that at one point or another. The reactor should have been shut down at that point in accordance with the requirements of Section 9 of the Operating Procedures. Not shutting down the reactor was presumably not incidental according to INSAG-7: "[t]he Commission assumes that the personnel deliberately violated [Section 9] requirement" (INSAG-7 1992, 71). Furthermore, after the accident, Kazachkov admitted that they knew the regulation that operating below the value of 15 ORM rods was forbidden and that the reactor should have been shut down immediately (Plokhy 2018, 9091). After 23:10 on the 25th of April, when the ORM measurement indicated a value equivalent with 26 inserted rods, during the process of the continuous power reduction, the operators constantly had to withdraw rods. Even if they did not know the exact value, they must have known that it was constantly decreasing (see also Fig. 1.). At least this information was available for them, so it would have been a reasonableexpectation for them to check the ORM values more regularly. But, according to the first historical reconstruction, that did not happen on the night in question. 


\subsubsection{Cumbersome accessibility}

INSAG-7 identifies some relevant and important circumstances that could have contributed to the operators' inclination towards negligence. First, as mentioned earlier, measuring the ORM was a complicated and time-costly process. The computer and instrument for measuring the ORM value were placed 50 metres away from the control console (INSAG-7 1992, 6, 72). Getting to the instrument, measuring the status of the rods, making the calculations, getting back to the control room, and getting the results of the measurement required 10-15 minutes (Ibid). This can be labelled as a problem of cumbersome accessibility.

\subsubsection{Temporal inaccessibility}

In addition, there is a note in the senior reactor control engineer's operating log claiming that "[t]he PRIZMA calculation code was found to be unreliable at this time, because it did not take into account the position of the rods of automatic regulators Nos 1, 2 and 3 (a total of 12 rods)" (INSAG-7 1992, 71). Hence, if the operators had intended to check the ORM values, they would have had to measure them manually in the core and then make non-linearity corrections. Whether it was possible in the actual energy range is uncertain though. If it was possible that negligent rule-violation was apparent: they should have regularly measured the ORM values manually. If it was not possible, then the ORM values would be temporarily inaccessible for the operators, creating a 'grey zone' because there was no regulation for such cases: "[t]he Operating Procedures and other operating documentation did not prescribe the actions to be taken by personnel under such circumstances (in the event of unreliable calculation) and similar circumstances (for example, in the event of complete failure of the PRIZMA code to determine the ORM)” (INSAG-7 1992, 71). This can be labelled as a problem of temporal inaccessibility.

These are the cases for which operators should have prepared especially carefully. These cases also reveal that the naïve conception of obedient or compliant rule-following is insufficient to provide adequate rule-following behaviour. There could be circumstances when it is somewhat cumbersome to acquire the relevant data that are required for determining whether a situation falls within the scope of a regulation (cumbersome accessibility problem), or when it is straightforwardly impossible to do so (temporal inaccessibility problem). These suggest that in addition to conscious and proactive self-regulation, proper rule-following also requires an understanding of the role of the instructions.

As we have seen so far, there were difficulties with measuring and controlling the ORM, and that can provide some excuse for the operators: if they could not access up-to-date ORM values constantly and easily, it becomes unrealistic to expect them to follow a rule related to those values. These difficulties could have contributed to the operators' inclination towards negligence, thereby providing further excuses for the rule-breaking. 


\subsection{The importance excuses}

In the previous section, we investigated the case scenario that the operators were not aware of the ORM values at the time of the accident. Now we shall see what consequences it would have had if they were not aware of the importance of those values. As mentioned, one of the historical reconstructions suggests that at the beginning of the test at 1:23:10, the operators knew the value of the ORM measured at 1:22:30, but not the danger of violating the ORM regulation this way (INSAG-7 1992, 78; Malko 2002, 19). How could that be the case? The answer is that the importance of the ORM rules was not clarified to them.

Regulations are never self-explanatory; they require interpretation. Reflections made by the IAEA in the INSAG-7 report confirmed the expectation that if the rule-follower does not understand the importance of a rule (he/she does not understand the function of the rule in the operation of the system, he/she is unaware of the possible consequences of violating that rule, etc.), this fact strengthens his/her inclination to violate the rule.

In the Chernobyl case, two problems with importance were apparent that can be taken as further excuses for the rule-breaking. First, the ORM regulations did not qualify as important rules. Some parameters were treated as important, while others were not, with the ORM parameters among the latter. This had an indirect consequence that the ORM regulations were considered less important than the other regulations. This possibly made it tempting for the operators to ignore them. Second, some important functions of the ORM were not explained in the regulations at all. The operators did not know the extreme importance of the ORM in emergencies. This led to a fatal misunderstanding of the role of the ORM. Let us look at these problems in detail.

\subsubsection{ORM and the important parameters list}

As we have seen in the previous section, it was not an easy task to keep the ORM values up to date. This could have also strengthened the operators' impression that the ORM was unimportant. As deputy chief engineer Anatoly Dyatlov claimed, "the operator has to perform up to 1000 manipulations per hour and monitor about 4000 parameters simultaneously. In these circumstances, it is somewhat cynical to accuse him of overlooking something" (Dyatlov 2006). Selecting some parameters was part of the job of operators so that they could focus on the parameters only which were important in that situation.

An obvious choice for what should be 'overlooked' (i.e. unselected) is those parameters that are not claimed to be important. The ORM was one of those. There was some sort of clarification of the importance of the regulations: a list in the Operating Procedures document distinguished several parameters so that the regulations connected to these parameters were taken as priorities. But the ORM was not included in this list (INSAG-7 1992, 72).

Even though post-factum discussion of the Chernobyl disaster regularly arrives at the ORM problem, this was not the case prior to the accident. When controlling 
the reactor at lower power levels, the operators were required to control approx. 1000 operations per person per hour (INSAG-7 1992, 5). The ORM was only one of the parameters to be monitored regularly, and it was even not claimed to be important. Its lack of inclusion in the list might also have implicitly suggested to the operators that the ORM was not important because it was not claimed to be important.

\subsubsection{The emergency function of the ORM}

But why was the ORM that important, and if it was, why was its importance underestimated? The ORM had two fundamental functions. First, it provided power control and manoeuvring potential by the insertion/withdrawal of rods. Second, it functioned as an emergency protection system that was suitable for instant safety actions when all the rods had been dropped into the core completely. The efficiency and significance of the second function were not clarified; however, the instructions explained explicitly the first function only (INSAG-7 1992, 72).

Hence, the operators were aware of how the ORM functioned under normal circumstances, but they were unaware of exactly how it functioned in an emergency. More precisely, it was not clarified that under certain circumstances, the role of the ORM was emergency protection, and hence its importance was dramatically increased under those circumstances. Since the ORM functions differed in normal cases from emergency cases, because, in low energy ranges, a low ORM value was an indicator of instability, the fact that the operators were not prepared for receiving this indication could, and did, result in dramatic consequences.

To sum up the importance excuses, the operators' breaking of the ORM regulations created an emergency, and their lack of knowledge that the ORM also functioned as an emergency protection tool further increased the chances of an accident. Hence, unimportance is also a twofold excuse for the operators' disregarding the ORM regulations for at least three reasons. First, they were not informed that the ORM regulations were important regulations. Second, they were indirectly informed that the ORM regulations were straightforwardly unimportant. Third, they were not informed that the ORM regulations were especially important (in fact, crucial) in emergency cases created by disregarding the ORM regulations. This does not release them from violating the regulations, but better informing them about the importance of the ORM could have prevented the disaster. Rule-makers may expect blind rule-following, but in order to be super-safe, they must also consider rule-breaking. Making explicit which rules are absolutely unbreakable is a necessary step in narrowing the logical space of possible excuses that might pseudo-rationalise rule violation.

\section{Consequences Generalised}

The above-mentioned six excuses derived from INSAG-7 and other historical resources explicate the operators' possible rationalisations for violating the ORM rules. These violations, even if not triggered, significantly contributed to the Cher- 
nobyl accident. Discussing these excuses does not serve to objectively rationalise the operators' acts, and our goal was not their exculpation, or to transfer responsibility to the designers either. Our aim was to understand the operators' potential internal defence mechanisms that could serve as subjective grounds for the rationalisation of what they presumably intended to do and what they finally did. As rationalisations, these excuses contributed to the disaster. In light of INSAG-7, in order to avoid future disasters, understanding and properly managing them is necessary. Regulations must reflect on these (and similar) excuses and find ways of preventing their occurrence, whether they are objectively rational or not.

A reason for the necessity of reflection is that even if the excuses were not objectively rational, they contributed to decreasing the subjective irrationality of breaking the rules. In order to make the rationalisation mechanism explicit, we provide a reconstruction of these excuses as potential premises for an argument.

The 'no chance of an accident' excuses

(E1) The reactor is fail-safe.

(E2) ORM rule violations happened several times before with no consequences.

(NE) ORM rules can be violated with no negative consequences.

The accessibility excuses

(E3) ORM values are cumbersome to access.

(E4) ORM values are sometimes temporarily inaccessible.

(AE) ORM values are not easy to keep up to date in order to comply with the ORM rules.

The importance excuses

(E5) ORM values are not included in the important parameters list.

(E6) ORM values are believed to be unimportant in emergencies.

(IE) ORM rules are not as important as the other rules.

From (NE\&AE\&IE), operators could conclude that

(C) Violating ORM rules is excusable.

(E1 \& E2), i.e. the myth of fail-safety along with inductive generalisation supported (NE) the false belief that there was no chance of an accident, and hence it was excusable to violate the rules because no serious problem could occur from that violation (see E1), and furthermore, the same rules had been violated earlier with no serious consequences (see E2). Both (E1) and (E2) could be separately sufficient for serving as a reason for breaking the ORM rules, but as demonstrated, they also mutually supported one another.

Preventive principles can build on an analysis of the social environment in the case of (E1), and the operation history in the case of (E2). It could be constantly investigated whether technological features communicated externally in the wider social environment interfere with operating rules. A conflict between the image communicated and the real technological background can result in myths, like that 
of fail-safety in the case of Chernobyl. Preceding events in the operating history that could lead to a loosening of discipline can also be detected and responded to accordingly. This could help prevent the spread of worst practice.

(E3 \& E4), i.e. problems that made access to the ORM values cumbersome (see E3) or temporarily impossible (see E4) supported (AE), the claim that ORM values were not easy to keep up to date in order to follow the rule, and hence ignoring them could seem as a practically viable option for operators. (E3) and (E4) provide excuses for a negligent violation that does not seem to be rule-breaking because of its indirectness, and hence it may seem to be more excusable than direct violation, making it more dangerous as even agents fully committed to rule-following can negligently violate rules through a lack of knowledge of the relevant parameters.

From (E3) and (E4), it can be seen that operational circumstances play a central role in rule-following. Operational circumstances are not the only factor that can prevent operators from rule-following. It can also be sufficient if some information relevant for rule-following is hard to access, or is temporarily inaccessible. These can also raise the chances of a severe negligence as it may incline the operators to ignore information that is not suitably accessible. To prevent situations like this, it must be investigated whether operational circumstances support or cumber operations and whether access can be established for all relevant information in all possible cases.

Finally, from (E5 \& E6), (IE) follows, i.e. ORM values were not important because they were not claimed to be important (see E5), and the operators did not know that they were especially important in an emergency (see E6). In order to incline towards rule-following behaviour, it is often not sufficient if a rule only ascertains what to do. In the Chernobyl case, knowing the importance of the ORM values was proved to be a prerequisite for following the ORM regulations (E5) (INSAG-7 1992, 14-15). Knowing the role of the ORM in an emergency may be unimportant in ordinary cases, but crucial insofar as the ORM regulations still be broken. This made the expectation that operators must follow the rule circular: they were not expected to know the reasons and motivations behind the rules because, on the condition that they do not break the rule, they do not need that knowledge, but unknowing the consequences can or could make the rule-breaking subjectively excusable.

Prevention in these cases can be grounded in a proper mediation of the designer's knowledge and operative knowledge. A revision of the operating rules and regulations, including their coherence, form and content, and their actual wording can bring unintentional underestimations of some rules (E5), or an incompleteness of the content to be transferred by the regulations to the operators in terms of the purpose(s) the rules serve (E6) to surface.

Such preventions are necessary for the following (practical) reasons. It is clear from INSAG-7 that rule-following behaviour, such as compliance or obedience, is a necessary expectation from operators (INSAG-7 1992, 30, 79). But the report also suggests that the regulation based on this expectation was insufficient. The operators did not comply with the regulation, even though INSAG-7 reports that they were "fairly typical, mature and stable group of specialists with qualifications regarded in the USSR as satisfactory. They were no better, but no worse, than the personnel at 
other nuclear plants" (INSAG-7 1992, 30). Based on the analyses of the Prognoz Psychological Research Laboratory of the USSR Ministry of Nuclear Power and Industry, INSAG-7 concludes that "personnel at the Chernobyl plant did not have any extraordinary characteristics" and hence their personalities "were not such as to have been a direct cause of the accident” (INSAG-7 1992, 30). Since the operators' inclination not to comply with the regulation did not depend on some irregular psychological conditions, their behaviours should be rationally interpreted. This means that the widely accepted conception of rule-following as (com)pliance or obedience is to be taken as a necessary but insufficient one if we really want to understand the actual behaviour of the operators. Extending the conceptual framework by the concept of excuses can also help to anticipate as well as to prevent the rule-breaking behaviour of operators. ${ }^{1}$

Complexity is a further important issue we have touched upon. In a complex situation like operating a nuclear reactor, complexity occurs not only at the level of the operations but in the conditions of rule-following as well. Under complexity, compliance or obedience becomes an insufficient condition for rule-following: our rule-interpretive environment is rich, and compliance is just one of many segments of it. Understanding the rule, putting it into the context of the whole system of regulations (see esp. the importance excuses), into technological (the accessibility excuses), and the social environment (the myth of fail-safety) are also constitutive elements of how we relate ourselves to rules, as well as the consequences of our earlier rule-following behaviour (the inductive generalisation excuse). In order to understand such complexities and possible reactions of agents to unforeseen situations, rationalisations or defence mechanisms must be supposed in the background. As a further step, for preventive reasons, a possibility of such excuses must be avoided once they are recognised. Knowing possible excuses for rule violation can help prepare agents to resist inclinations towards subjective defence mechanisms that naturally occur in human thinking and behaviour, especially in sharp situations like operating a nuclear reactor.

\footnotetext{
${ }^{1}$ There are frameworks for extending the conception of rule-following as (com)pliance or obedience (see esp. Törneke, Luciano, and Valdivia-Salas 2008; Peláez 2013; and Kissi et. al 2017), though the application of them to the Chernobyl case is not straightforward.
} 


\section{References}

Abramova, V. N. "What Needs to be Changed Based on Lessons Learned from Chernobyl." In Human And Organizational Aspects Of Assuring Nuclear Safety - Exploring 30 Years Of Safety Culture, 81-100. Vienna, Austria: International Atomic Energy Agency, 2019. Accessed December 3, 2021.

https://www-pub.iaea.org/MTCD/Publications/PDF/P1810 web.pdf

Nuclear Engineering International. Dyatlov, Anatoly. "How it was: an operator's perspective.”Accessed May 14, 2021.

https://www.neimagazine.com/features/featurehow-it-was-an-operator-s-perspective/

Hultman, Nathan, and Jonathan Koomey. “Three Mile Island: The driver of US nuclear power's decline?” Bulletin of the Atomic Scientists 69, no. 3 (2013): 63-70.

doi: $10.1177 / 0096340213485949$

IAEA-TECDOC-1329. Safety Culture in Nuclear Installations: Guidance For Use in the Enhancement of Safety Culture. Operation Safety Section, International Atomic Energy Agency, Vienna, 2002. Accessed December 3, 2021.

https://www-pub.iaea.org/MTCD/Publications/PDF/te_1329_web.pdf

INSAG-7. The Chernobyl Accident: Updating of INSAG-1. A Report by the International Nuclear Safety Advisory Group. Safety Series No. 75-Insag-7. International Atomic Energy Agency, Vienna, 1992. Accessed May 11, 2021.

https://www-pub.iaea.org/MTCD/publications/PDF/Pub913e_web.pdf

INSAG-15. Key Practical Issues in Strengthening Safety Culture. A Report by the International Nuclear Safety Advisory Group. International Atomic Energy Agency, Vienna, 2002. Accessed December 3, 2021.

https://www-pub.iaea.org/MTCD/Publications/PDF/Pub1137_scr.pdf

Kissi, Ama, Sean Hughes, Gaëtan Mertens, Dermot Barnes-Holmes, Jan De Houwer, and Geert Crombez. “A Systematic Review of Pliance, Tracking, and Augmenting.” Behavior Modification 41, no. 5 (2017): 683-707.

https://doi.org/10.1177/0145445517693811

Plokhy, Serhii. Chernobyl: History of a Tragedy. New York: Basic Books, 2018.

Malko, Mikhail V. "The Chernobyl Reactor: Design Features and Reasons for Accident.” In Recent Research Activities about the Chernobyl NPP Accident in Belarus, Ukraine and Russia, edited by Tetsuji Imanaka, 11-27. Japan: Kyoto University Research Reactor Institute, 2002. http://large.stanford.edu/courses/2014/ph241/alnoaimi2/docs/kr79.pdf

Medvedev, Zhores A. The Legacy of Chernobyl. New York: W. W. Norton \& Companyk, 1990.

Niklas, Törneke, Luciano Carmen, and Sonsoles Valdivia-Salas. "Rule-Governed Behavior and Psychological Problems.” International Journal of Psychology and Psychological Therapy 8, no. 2 (2008): 141-156.

https://www.ijpsy.com/volumen8/num2/191/rule-governed-behavior-and-psychologicalEN.pdf

Pelaez, Martha. "Dimensions of Rules and Their Correspondence to Rule-Governed Behavior.” European Journal of Behavior Analysis 14, no. 2 (2013): 259-270. DOI: $10.1080 / 15021149.2013 .11434459$

Szathmáry, Zoltán, and Attila, Aszódi. Csernobil: Tények, okok, hiedelmek. Budapest: Typotex Kiadó: Budapest, 2005. 